\title{
Cost Allocation and Convex Data Envelopment
}

Hougaard, Jens Leth; Tind, Jørgen

Publication date:

2008

Document version

Publisher's PDF, also known as Version of record

Citation for published version (APA):

Hougaard, J. L., \& Tind, J. (2008). Cost Allocation and Convex Data Envelopment. Department of Economics, University of Copenhagen. 


\section{Discussion Papers Department of Economics University of Copenhagen}

No. $08-02$

Cost Allocation and Convex Data Envelopment

Jens Leth Hougaard

Jørgen Tind

Studiestræde 6, DK-1455 Copenhagen K., Denmark

Tel. : +4535323082 - Fax: +4535323000

http://www.econ.ku.dk

ISSN: 1601-2461 (online) 


\title{
Cost Allocation and Convex Data Envelopment
}

\author{
Jens Leth Hougaard \\ Department of Economics \\ University of Copenhagen \\ Jørgen Tind \\ Department of Mathematical Sciences \\ University of Copenhagen
}

January, 2008

\begin{abstract}
This paper considers allocation rules. First, we demonstrate that costs allocated by the Aumann-Shapley and the Friedman-Moulin cost allocation rules are easy to determine in practice using convex envelopment of registered cost data and parametric programming. Second, from the linear programming problems involved it becomes clear that the allocation rules, technically speaking, allocate the non-zero value of the dual variable for a convexity constraint on to the output vector. Hence, the allocation rules can also be used to allocate inefficiencies in non-parametric efficiency measurement models such as Data Envelopment Analysis (DEA). The convexity constraint of the BCC model introduces a non-zero slack in the objective function of the multiplier problem and we show that the cost allocation rules discussed in this paper can be used as candidates to allocate this slack value on to the input (or output) variables and hence enable a full allocation of the inefficiency on to the input (or output) variables as in the CCR model.
\end{abstract}

Keywords: Cost Allocation, Convex Envelopment, Data Envelopment Analysis, Slack Allocation. 
Correspondence: Jens Leth Hougaard, Department of Economics, University of Copenhagen, Studiestraede 6, 1455 Copenhagen K., Denmark.

E-mail: Jens.Leth.Hougaard@econ.ku.dk

Acknowledgements: The authors acknowledge financial support from the Danish Social Science Research Council. 


\section{Introduction}

Production of multiple goods often involves joint costs, which complicate internal cost allocation. The literature on cost allocation has mainly focused on two cost allocation rules in such cases,

i. the Aumann-Shapley rule introduced in Aumann and Shapley (1974), which can be seen as a multi dimensional version of average cost sharing. Axiomatic characterizations are e.g. given (independently) in Billera and Heath (1982) and Mirman and Tauman (1982). Billera, Heath and Raanan (1978) and Samet, Tauman and Zang (1983) are early examples of application.

ii. the Friedman-Moulin rule that was recently introduced in Friedman and Moulin (1999) and can be seen as a multi dimensional version of serial cost sharing. Friedman and Moulin also provide an axiomatic characterization.

These rules have many appealing properties in common. As demonstrated in Friedman (2003) they are both members of a class consisting of convex combinations of path generated rules. This class is characterized by the standard properties of additivity and separability. However, there are also properties which separates the two. Friedman and Moulin (1999) show that the Aumann-Shapley rule does not satisfy demand monotonicity in the sense that if the level of some output increases this output will also receive a larger cost share (demand monotonicity is satisfied by the Friedman-Moulin rule). On the other hand, the Friedman-moulin rule does not satisfy simple scale invariance (as do the Aumann-Shapley rule).

The aim of the present paper is two-fold: First, we shall demonstrate that the cost shares of both rules are easy to determine in practice. Both rules require the definition of a cost function and we suggest to estimate the cost function using simple convex envelopment of observed cost data. With this approach we are able to use parametric programming to determine the cost shares related to arbitrary output vectors.

Second, from the linear programming problems involved it becomes clear that the allocation rules, technically speaking, allocate the non-zero value of the dual variable for a convexity constraint on to the output vector. Hence, there is a flip side to the story of cost allocation, which concerns the allocation of inefficiencies in non-parametric efficiency measurement models such as Data Envelopment Analysis (DEA) see e.g. Cooper, Seiford and Zhu (2004). In the original DEA model using constant returns to scale (the CCR model) the efficiency score of the envelopment problem can be seen as allocated on to 
the input (or output) variables via the dual multiplier problem. Adding the convexity constraint in the variable returns to scale version of DEA (the BCC model) introduces a non-zero slack in the objective function of the multiplier problem and we demonstrate that the cost allocation rules discussed in this paper can be used as candidates to allocate this slack value on to the input (or output) variables and hence enable a full allocation of the inefficiency on to the input (or output) variables like in the case of the CCR model.

Papers by Cook and Kress (1999), Cook and Zhu (2005) and Beasley (2003) also deal with cost allocation using non-parametric techniques, but in a different context than the present paper. They consider the case where a group of similar production units, each using the same type of multiple inputs to produce the same type of multiple outputs (e.g. branches of a bank or other types of similar subunits), are operating under a common fixed cost (e.g. marketing costs), which has to be allocated among the units in the group. Hence, costs are not directly a function of the inputs and outputs involved, but constitutes a fixed amount that has to be allocated according to the multidimensional production characteristics of all subunits.

The paper is organized as follows: Section 2 introduces the cost allocation model and the Aumann-Shapley and Friedman-Moulin cost allocation rules are defined. Moreover, it is shown how the cost function can be estimated empirically using convex envelopment of cost data. Section 3 establishes the parametric programming problems which are relevant for the calculation of cost shares according to the Aumann-Shapley and Friedman-Moulin cost allocation rules and illustrates the procedure by a simple example. In Section 4 it is demonstrated that such cost allocation rules can be used to allocate inefficiencies on to specific inputs (or outputs) in the DEA framework (or to be more precise, in the BCC model). Section 5 closes with final remarks.

\section{The Cost Allocation Model}

Consider $n$ different types of outputs and let $q \in \mathbf{R}_{+}^{n}$ be a (non-negative) output vector where $q_{i}$ is the level of output $i$. The cost of producing any (output) vector $q$ is given by a non-decreasing cost function $C: \mathbf{R}_{+}^{n} \rightarrow \mathbf{R}$ where $C(0)=0$ (i.e. no fixed costs).

Denote by $(q, C)$ a cost allocation problem and let $\phi$ be a cost allocation rule, which specifies a unique vector of costs related to each output $x=$ $\left(x_{1}, \ldots, x_{n}\right)=\phi(q, C)$ where

$$
\sum_{i=1}^{n} x_{i}=C(q)
$$


and $x_{i}$ is the cost related to output $i$.

In particular, consider the set of continuously differentiable cost functions $C$ and denote by $\partial_{i} C(q)$ the first order derivative of $C$ at $q$ with respect to the $i$ th argument.

Define the Aumann-Shapley rule $\phi^{A S}$ (Aumann and Shapley 1974) by allocated costs,

$$
x_{i}^{A S}(q, C)=\int_{0}^{q_{i}} \partial_{i} C\left(\frac{t}{q_{i}} q\right) d t=q_{i} \int_{0}^{1} \partial_{i} C(t q) d t \text { for all } i=1, \ldots, n .
$$

Note, that $\sum_{i \in N} x_{i}^{A S}(q, C)=C(q)$. In particular, $p_{i}^{A S}=\int_{0}^{1} \partial_{i} C(t q) d t$ can be seen as the unit cost of output $i$ - also known as the Aumann-Shapley price.

\section{Example 1}

Consider the two-outputs case $n=2$ and let costs be determined by the cost function

$$
C(q)=q_{2}+\left(q_{1}+q_{2}\right)^{0.5}
$$

If, for instance, the output vector is given by $q=\left(q_{1}, q_{2}\right)=(1,2)$ the total cost is $C(1,2)=2+3^{0.5}=3.73$. Since,

$$
\partial_{1} C(q)=0.5\left(q_{1}+q_{2}\right)^{-0.5} \text { and } \partial_{2} C(q)=1+0.5\left(q_{1}+q_{2}\right)^{-0.5}
$$

the Aumann-Shapley allocated costs are given by,

$$
x_{1}^{A S}((1,2), C)=\int_{0}^{1} 0.5(3 t)^{-0.5} d t=\left[3^{-0.5} t^{0.5}\right]_{0}^{1}=0.58
$$

and

$$
x_{2}^{A S}((1,2), C)=2 \int_{0}^{1}\left(1+0.5(3 t)^{-0.5}\right) d t=2\left[t+3^{-0.5} t^{0.5}\right]_{0}^{1}=3.15,
$$

with corresponding unit costs (Aumann-Shapley prices) of 0.58 and 1.58 respectively.

Now, consider a given output vector $q$ where the outputs are labeled according to increasing quantities, i.e. $q_{1} \leq \ldots \leq q_{n}$. Define the Friedman-Moulin rule $\phi^{F M}$ (Friedman and Moulin 1999) by the allocated costs

$$
x_{i}^{F M}(q, C)=\int_{0}^{q_{i}} \partial_{i} C((t e) \wedge q) d t, \quad \text { for all } i=1, \ldots, n,
$$


where $e=(1, \ldots, 1)$ and $(a \wedge b)_{i}=\min \left\{a_{i}, b_{i}\right\}$. Note, that $\sum_{i \in N} x_{i}^{F M}(q, C)=$ $C(q)$. The allocated cost, $x_{i}^{F M}$, is determined as the integral of the marginal cost $\partial_{i} C$ along a path joining 0 and $q$ by raising all coordinates at the same speed and freezing a coordinate once it reaches $q_{i}$ - that is, by the path: $0 \rightarrow\left(q_{1}, \ldots, q_{1}\right) \rightarrow\left(q_{1}, q_{2}, \ldots, q_{2}\right) \rightarrow\left(q_{1}, q_{2}, q_{3}, \ldots, q_{3}\right) \rightarrow \ldots \rightarrow\left(q_{1}, \ldots, q_{n}\right)$.

\section{Example 2}

Using again the cost function

$$
C(q)=q_{2}+\left(q_{1}+q_{2}\right)^{0.5}
$$

with partiel derivatives

$$
\partial_{1} C(q)=0.5\left(q_{1}+q_{2}\right)^{-0.5} \text { and } \partial_{2} C(q)=1+0.5\left(q_{1}+q_{2}\right)^{-0.5}
$$

we now get allocated costs

$$
\begin{aligned}
x_{1}^{F M}((1,2), C) & =\int_{0}^{1} \partial_{1} C(t, t) d t=\int_{0}^{1} 0.5(2 t)^{-0.5} d t \\
& =\left[2^{-0.5} t^{0.5}\right]_{0}^{1}=0.71
\end{aligned}
$$

and

$$
\begin{aligned}
& x_{2}^{F M}((1,2), C)=\int_{0}^{1} \partial_{2} C(t, t) d t+\int_{1}^{2} \partial_{2} C(1, t) d t \\
= & \int_{0}^{1}\left(1+0.5(2 t)^{-0.5}\right) d t+\int_{1}^{2}\left(1+0.5(1+t)^{-0.5}\right) d t \\
= & {\left[t+2^{-0.5} t^{0.5}\right]_{0}^{1}+\left[t+(1+t)^{0.5}\right]_{1}^{2}=3.02 . }
\end{aligned}
$$

In practice, of course, the cost function has to be estimated on the basis of registered cost data. Below, we shall consider a non-parametric estimation of $C$.

\subsection{Data Envelopment}

Let $\left\{\left(q_{j}, C_{j}\right)\right\}_{j=1, \ldots, h}$ be a set of $h$ observations of (output) vectors $q_{j}$ and their associated production cost $C_{j}$. These observations can be construed as originating either from the same firm over $h$ time periods or from $h$ different firms at a given point in time.

Based on such a data set a cost function may be estimated empirically using a traditional parametric approach where a given functional form is 
postulated and parameters associated with this form are estimated. This approach is taken in numerous studies of cost functions.

However, here we suggest to consider a non-parametric approach where the production possibility set is estimated using simple convex envelopment of the data points as suggested in Charnes, Cooper and Rhodes (1978) and further treated in Banker, Charnes and Cooper (1984) and Banker and Maindiratta (1988).

We may consider costs $C(q)$ as the result of producing output vector $q$. Hence, a 'cost' possibility $z$ is a data point $(q, C) \in \mathbf{R}_{+}^{n} \times \mathbf{R}_{+}$where $C$ is the possible cost associated with producing $q$. Denote by $\mathcal{C} \subset \mathbf{R}^{n+1}$ the cost possibility set.

Following Banker, Charnes and Cooper (1984) we assume,

Convexity: If $z, z^{\prime} \in \mathcal{C}$ then $\lambda z+(1-\lambda) z^{\prime} \in \mathcal{C}$ for $\lambda \in[0,1]$

Decreasing returns: If $z \in \mathcal{C}$ then $\lambda z \in \mathcal{C}$ for $\lambda \in[0,1]$.

Free disposability: Let $z=(q, C) \in \mathcal{C}$ and let $q^{\prime} \leq q$ and $C^{\prime} \geq C$ then $z^{\prime}=\left(q^{\prime}, C^{\prime}\right) \in \mathcal{C}$.

For a given data set $\left\{\left(q_{j}, C_{j}\right)\right\}_{j=1, \ldots, h} \in\left\{\mathbf{R}^{n+1}\right\}^{h}$ we obtain an empirical estimate $\mathcal{C}^{*}$ of the cost possibility set $\mathcal{C}$ as the intersection of sets satisfying the three conditions above, which contains all the data points, i.e. as,

$$
\mathcal{C}^{*}=\left\{(\hat{q}, \hat{C}) \in \mathbf{R}_{+}^{n+1} \mid \sum_{j=1}^{h} \lambda_{j} q_{j} \geq \hat{q}, \sum_{j=1}^{h} \lambda_{j} C_{j} \leq \hat{C}, \sum_{j=1}^{h} \lambda_{j} \leq 1, \lambda_{j} \geq 0, \forall j\right\}
$$

Moreover, let

$$
\mathcal{Q}^{*}=\left\{q \mid \exists \bar{C}:(q, \bar{C}) \in \mathcal{C}^{*}\right\}
$$

be the set of possible productions $q$ given the observed data set.

Alternatively we may replace the assumption of decreasing returns (in production) with the assumption:

Constant returns: If $z \in \mathcal{C}$ then $\lambda z \in \mathcal{C}$ for $\lambda \geq 0$.

In this case we get

$$
\mathcal{C}^{*}=\left\{(\hat{q}, \hat{C}) \in \mathbf{R}_{+}^{n+1} \mid \sum_{j=1}^{h} \lambda_{j} q_{j} \geq \hat{q}, \sum_{j=1}^{h} \lambda_{j} C_{j} \leq \hat{C}, \lambda_{j} \geq 0, \forall j\right\} .
$$


In the following we shall use the assumption of decreasing returns and only use the case of constant returns for comparison.

The (efficient) boundary of $\mathcal{C}^{*}$ is a non-parametric estimate of the cost function $C(q)$. Note that due to convexity of $\mathcal{C}^{*}$ the estimated cost function $C^{*}(q)$ will be convex and piecewise linear. The convexity assumption may be relaxed (see e.g. Bogetoft 1996, Bogetoft, Tama and Tind 2000) but, for the present purpose and the ease of exposition, we continue with the convex version above.

Even though the estimate $C^{*}$ is not differentiable in general, it is continuosly differentiable along a line segment $[0, z]$, except perhaps for finitely many points (see e.g Samet, Tauman and Zang 1984). Thus, both the AumannShapley and the Friedman-Moulin rule are well defined with respect to the estimate $C^{*}$.

\section{Cost Allocation Using Data Envelopment}

First consider calculation of the Aumann-Shapley cost shares with respect to the estimate $C^{*}$. In connection with transportation problems, Samet, Tauman and Zang (1984) suggested to use parametric programming (see e.g. Bazaraa, Jarvis and Sherali 1990) to determine the Aumann-Shapley cost shares. We use the same technique to determine the Aumann-Shapley prices, which are easily found as a finite sum of gradients of the linear pieces of $C^{*}$ along the line segment $[0, q]$ weighted with the normalized length of the subintervals where $C^{*}$ has constant gradient.

Select a given output vector $\hat{q} \in \mathcal{Q}^{*}$. In our case, we first consider parameter values $t \in[0,1]$ and solve

$$
\begin{array}{ll}
\min & \sum_{j=1}^{h} \lambda_{j} C_{j} \\
\text { s.t. } & \sum_{j=1}^{h} \lambda_{j} q_{j} \geq t \hat{q} \\
& \sum_{j=1}^{h} \lambda_{j} \leq 1 \\
& \lambda_{j} \geq 0, \forall j .
\end{array}
$$

As a result we get the relevant subintervals of $[0, \hat{q}]$ for which the gradients are constant, i.e. a series of values $t_{m}$ for which the gradient is constant on the interval $\left[t_{m-1}, t_{m}\right]$. The values of the gradients are equal to the values of 
the optimal dual variables corresponding to the constraints (4). The optimal dual variable corresponding to the convexity constraint (5) is usually nonzero. In technical terms, we may consider the Aumann-Shapley allocation procedure as a way to transfer and allocate the value of this dual variable to the dual variables corresponding to (4) as illustrated in Example 3 below.

If the convexity constraint (5) is removed, we get a constant returns to scale version of the problem, i.e.

$$
\begin{array}{ll}
\min & \sum_{j=1}^{h} \lambda_{j} C_{j} \\
\text { s.t. } & \sum_{j=1}^{h} \lambda_{j} q_{j} \geq t \hat{q} \\
& \lambda_{j} \geq 0, \forall j .
\end{array}
$$

In this model the gradients are the same all over the interval $[0, \hat{q}]$ with values determined by the optimal dual variables, and by linear programming duality they can be applied directly in the cost sharing problem (as Aumann-Shapley prices).

\section{Example 3}

Consider the following data from four observations:

\begin{tabular}{|l|l|l|l|}
\hline Obs. & $q_{1}$ & $q_{2}$ & $C$ \\
\hline 1 & 2 & 3 & 6 \\
2 & 3 & 1 & 5 \\
3 & 4 & 5 & 15 \\
4 & 5 & 2 & 10 \\
\hline
\end{tabular}

Let $\hat{q}=(4,3)$ and define $\bar{q}=\left(\bar{q}_{1}(t), \bar{q}_{2}(t)\right)=\left(\hat{q}_{1} t, \hat{q}_{2} t\right)=(4 t, 3 t)$. Hence, we solve the following problem:

$$
\begin{aligned}
& \min 6 \lambda_{1}+5 \lambda_{2}+15 \lambda_{3}+10 \lambda_{4} \\
& 2 \lambda_{1}+3 \lambda_{2}+4 \lambda_{3}+5 \lambda_{4} \geq 4 t \\
& 3 \lambda_{1}+1 \lambda_{2}+5 \lambda_{3}+2 \lambda_{4} \geq 3 t \\
& \lambda_{1}+\lambda_{2}+\lambda_{3}+\lambda_{4} \leq 1 \\
& \lambda_{i} \geq 0 \text { for } i=1, \ldots, 4 \text {. }
\end{aligned}
$$

By parametric linear programming we get, omitting the $t$ argument in the $\bar{q}$ variables: 


\begin{tabular}{|l|l|}
\hline$t$ & objective function \\
\hline $0 \leq t \leq \frac{7}{11}=0.64$ & $\frac{9}{7} \bar{q}_{1}+\frac{8}{7} \bar{q}_{2}$ \\
$\frac{7}{11} \leq t \leq \frac{11}{13}=0.85$ & $\frac{9}{5} \bar{q}_{1}+\frac{7}{5} \bar{q}_{2}-\frac{9}{5}$ \\
$\frac{11}{13} \leq t \leq 1$ & $\frac{17}{8} \bar{q}_{1}+\frac{19}{8} \bar{q}_{2}-\frac{43}{8}$ \\
\hline
\end{tabular}

From the above table we get Aumann-Shapley allocated costs

$$
x_{1}^{A S}=4 \times\left\{\frac{9}{7} \times \frac{7}{11}+\frac{9}{5} \times\left(\frac{11}{13}-\frac{7}{11}\right)+\frac{17}{8} \times\left(1-\frac{11}{13}\right)\right\}=6.09
$$

and

$$
x_{2}^{A S}=3 \times\left\{\frac{8}{7} \times \frac{7}{11}+\frac{7}{5} \times\left(\frac{11}{13}-\frac{7}{11}\right)+\frac{19}{8} \times\left(1-\frac{11}{13}\right)\right\}=4.16 .
$$

Observe that $x_{1}^{A S}+x_{2}^{A S}=10.25$ which is equal to the objective function value of the above program when $t=1$, as it should be.

The third convexity constraint is binding and receives in this case a nonzero dual variable value which is equal to the element $-\frac{43}{8}$ in the last row of the table. Again from the last row we see that the optimal dual variable corresponding to the first element in the output vector is equal to $\frac{17}{8}=2.125$. Multiplication of this price by the output quantity $\hat{q}_{1}=4$ gives the value of 8.50. The difference between $x_{1}^{A S}$ and this value is -2.41 . The similar difference corresponding to the second element of the output vector is -2.96 . The two differences add to $-5.37=-\frac{43}{8}$ which is equal to the value of the optimal dual variable corresponding to the convexity constraint, as it should be. In this way the dual variable for the convexity constraint is distributed on to the values of the output vector.

The corresponding constant returns to scale model, obtained by removal of the convexity constraint, is

$$
\begin{aligned}
\min \lambda_{1}+5 \lambda_{2}+15 \lambda_{3}+10 \lambda_{4} & \\
2 \lambda_{1}+3 \lambda_{2}+4 \lambda_{3}+5 \lambda_{4} & \geq 4 t \\
3 \lambda_{1}+1 \lambda_{2}+5 \lambda_{3}+2 \lambda_{4} & \geq 3 t \\
\lambda_{i} & \geq 0 \text { for } i=1, \ldots, 4
\end{aligned}
$$

In this case we get the same objective function for all $t$ as shown by the next table.

\begin{tabular}{|l|l|}
\hline$t$ & objective function \\
\hline $0 \leq t \leq 1$ & $\frac{9}{7} \bar{q}_{1}+\frac{8}{7} \bar{q}_{2}$ \\
\hline
\end{tabular}


The optimal dual variables for the two constraints of the program (7) are $\left(\frac{9}{7}, \frac{8}{7}\right)$ and they are equal to the coefficients of the objective function in the above table, as they should be. This confirms that the cost allocation by the Aumann-Shapley method in the case of constant return to scale is equivalent to a cost allocation based on the optimal dual variables of the model. In this case it is not necessery to allocate dual variables arising from an additional (convexity) constraint.

The same type of technique can be used to determine the allocated costs of the Friedman-Moulin rule. Consider an output vector $\hat{q} \in \mathcal{Q}^{*}$ and let $\hat{q}_{(\cdot)}=\left(\hat{q}_{(1)}, \ldots, \hat{q}_{(n)}\right)$ be the vector $\hat{q}$ where the indices have been permuted such that they are in increasing order, i.e. $\hat{q}_{(1)} \leq \ldots \leq \hat{q}_{(n)}$. Moreover, define vectors $p_{0}=(0, \ldots, 0), p_{1}=\left(\hat{q}_{(1)}, \ldots, \hat{q}_{(1)}\right), p_{2}=\left(\hat{q}_{(1)}, \hat{q}_{(2)}, \ldots, \hat{q}_{(2)}\right), \ldots$, $p_{i}=\left(\hat{q}_{(1)}, \ldots, \hat{q}_{(i-1)}, \hat{q}_{(i)}, \ldots, \hat{q}_{(i)}\right), \ldots, p_{n}=\hat{q}_{(\cdot)}$.

We now have to solve $n$ problems for $s=0, \ldots, n-1$, and parameter values $t \in[0,1]$,

$$
\begin{array}{ll}
\min & \sum_{j=1}^{h} \lambda_{j} C_{j} \\
\text { s.t. } & \sum_{j=1}^{h} \lambda_{j} q_{j} \geq p_{s}+t\left(p_{s+1}-p_{s}\right) \\
& \sum_{j=1}^{h} \lambda_{j} \leq 1 \\
& \lambda_{j} \geq 0, \forall j .
\end{array}
$$

Again we obtain the relevant subintervals of the path

$$
\left[p_{0}, p_{1}\right],\left[p_{1}, p_{2}\right], \ldots,\left[p_{n-1}, p_{n}\right]
$$

for which the gradients are constant, i.e. a series of values $t_{m}$ for which the gradient is constant on the interval $\left[t_{m-1}, t_{m}\right]$. We may then determine the gradients on all such subintervals and determine the Friedman-Moulin allocated costs as illustrated by the following example.

\section{Example 3 continued:}

Continuing the previous example we get that $\left(\hat{q}_{(2)}, \hat{q}_{(1)}\right)=\left(\hat{q}_{1}, \hat{q}_{2}\right)=(4,3)$, $p_{1}=(3,3)$ and $p_{2}=(3,4)$. Hence for $s=0$ we shall solve 


$$
\begin{aligned}
& \min 6 \lambda_{1}+5 \lambda_{2}+15 \lambda_{3}+10 \lambda_{4} \\
& 2 \lambda_{1}+3 \lambda_{2}+4 \lambda_{3}+5 \lambda_{4} \geq 3 t \\
& 3 \lambda_{1}+1 \lambda_{2}+5 \lambda_{3}+2 \lambda_{4} \geq 3 t \\
& \lambda_{1}+\lambda_{2}+\lambda_{3}+\lambda_{4} \leq 1 \\
& \lambda_{i} \geq 0 \text { for } i=1, \ldots, 4 \text {. }
\end{aligned}
$$

This gives us the table

\begin{tabular}{|l|l|}
\hline$t$ & objective function \\
\hline $0 \leq t \leq \frac{7}{9}=0.78$ & $\frac{9}{7} \bar{q}_{1}+\frac{8}{7} \bar{q}_{2}$ \\
$\frac{7}{9} \leq t \leq \frac{11}{12}=0.92$ & $\frac{9}{5} \bar{q}_{1}+\frac{7}{5} \bar{q}_{2}-\frac{9}{5}$ \\
$\frac{11}{12} \leq t \leq 1$ & $\frac{17}{8} \bar{q}_{1}+\frac{19}{8} \bar{q}_{2}-\frac{43}{8}$ \\
\hline
\end{tabular}

The contribution to $x_{1}^{F M}$ from this table is

$$
3 \times\left\{\frac{9}{7} \times \frac{7}{9}+\frac{9}{5} \times\left(\frac{11}{12}-\frac{7}{9}\right)+\frac{17}{8} \times\left(1-\frac{11}{12}\right)\right\}=4.281
$$

and to $x_{2}^{F M}$

$$
3 \times\left\{\frac{8}{7} \times \frac{7}{9}+\frac{7}{5} \times\left(\frac{11}{12}-\frac{7}{9}\right)+\frac{19}{8} \times\left(1-\frac{11}{12}\right)\right\}=3.843 .
$$

Next for $s=1$ we shall solve

$$
\begin{aligned}
& \min 6 \lambda_{1}+5 \lambda_{2}+15 \lambda_{3}+10 \lambda_{4} \\
& 2 \lambda_{1}+3 \lambda_{2}+5 \lambda_{3}+5 \lambda_{4} \geq 3+t \\
& 3 \lambda_{1}+1 \lambda_{2}+5 \lambda_{3}+2 \lambda_{4} \geq 3 \\
& \lambda_{1}+\lambda_{2}+\lambda_{3}+\quad \lambda_{4} \leq 1 \\
&
\end{aligned}
$$

implying the table

\begin{tabular}{|l|l|}
\hline$t$ & objective function \\
\hline $0 \leq t \leq 1$ & $\frac{17}{8} \bar{q}_{1}+\frac{19}{8} \bar{q}_{2}-\frac{43}{8}$ \\
\hline
\end{tabular}

The contribution to $x_{1}^{F M}$ is

$$
1 \times \frac{17}{8} \times 1=2.125
$$

and to $x_{2}^{F M}$

$$
0 \times \frac{19}{8} \times 1=0 .
$$

Hence, in total, the Friedman-Moulin allocated costs are given by

$$
x_{1}^{F M}=4.281+2.125=6.41
$$


and

$$
x_{2}^{F M}=3.843+0=3.84 \text {. }
$$

Again $x_{1}^{F M}+x_{2}^{F M}=10.25$ as it should be. So the Friedman-Moulin rule allocates more costs to $q_{1}$ in comparison to the Aumann-Shapley rule. Again we see that the value of the dual variable of the convexity constraint $-\frac{43}{8}=$ -5.37 is allocated to the values of the output vector, but this time with the shares -2.09 and -3.28 respectively for output 1 and output 2 .

\section{Allocation of efficiency in DEA}

The previous section treated the distribution of costs in a cost-output space subject to assumptions about convexity and disposability, which are standard in Data Envelopment Analysis (DEA). The purpose of this section is to use a similar type of approach with respect to the DEA model itself in production space. This is quite natural since basically costs can be considered as aggregated inputs producing output vector $q$. Hence, instead of estimating a cost function we now estimate a production function non-parametrically. Consequently, the observations are now inputs and outputs from a set of similar decision making units (DMU's) and the DEA model calculates an efficiency score for a selected DMU relative to a set of observed DMU's.

We shall here discuss the output oriented model, in which the output vector of the selected DMU is (radially) scaled to the largest extent given the DMUs' input use. The size of this scaling $(\theta)$ is the efficiency score assigned to the DMU.

Let $H$ denote the index set of all DMUs and let $\left(x_{j}, y_{j}\right)$ denote the inputoutput vector of the $j$ th DMU, which may contain both multiple inputs and multiple outputs. Specifically let $\left(x_{0}, y_{0}\right)$ be the input-output vector of the DMU under consideration. Then the standard output oriented constant returns to scale model (the CCR model) can be formulated as the following linear programming (envelopment) problem.

$$
\begin{array}{cl}
\max & \theta \\
\text { s.t. } & -\sum_{j \in H} \lambda_{j} y_{j}+\theta y_{0} \leq 0 \\
& \sum_{j \in H} \lambda_{j} x_{j} \leq x_{0} \\
& \lambda_{j} \geq 0 \text { for all } j \in H .
\end{array}
$$

The dual (multiplier) problem is

$$
\min u x_{0}
$$




$$
\begin{array}{ll}
\text { s.t. } & u x_{j}-v y_{j} \geq 0 \\
& u y_{o}=1 \\
& u, v \geq 0 .
\end{array}
$$

The dual variables in vector $u$ indicate the marginal value of the inputs for the determination of the efficiency score $\theta$. The total marginal value of all inputs is thus $u x_{0}$ which (by duality) equals $\theta$. Thus, looking at the multiplier problem we are able to allocate the inefficiency to each specific input category.

However, adding a convexity constraint as in the BCC model with variable returns to scale we get the following linear programming problem.

$$
\begin{array}{cl}
\max & \theta \\
\text { s.t. } & -\sum_{j \in H} \lambda_{j} y_{j}+\theta y_{0} \leq 0 \\
& \sum_{j \in H} \lambda_{j} x_{j} \leq x_{0} \\
& \sum_{j \in H} \lambda_{j}=1 \\
& \lambda_{j} \geq 0 \text { for all } j \in H .
\end{array}
$$

with the dual multiplier problem

$$
\begin{array}{cl}
\min & u x_{0}+u_{0} \\
\text { s.t. } & u x_{j}-v y_{j}+u_{0} \geq 0 \\
& u y_{o}=1 \\
& u, v \geq 0 \text { and } u_{0} \text { free. }
\end{array}
$$

Again the total marginal value of all inputs is thus $u x_{0}$ but in this case the amount differs from the value of the efficiency score by the amount $u_{0}$. The analogy with the cost sharing model now becomes clear since we may distribute $u_{0}$ onto the individual input elements by either the Aumann-Shapley approach or the Friedman Moulin approach in order to fully allocate the inefficiency to all inputs.

For example, using the Aumann-Shapley approach we get the following parametric program in the variable $t \in[0,1]$

$$
\begin{array}{cl}
\max & \theta \\
\text { s.t. } & -\sum_{j \in H} \lambda_{j} y_{j}+\theta y_{0} \leq 0 \\
& \sum_{j \in H} \lambda_{j} x_{j} \leq t x_{0}
\end{array}
$$




$$
\begin{aligned}
& \sum_{j \in H} \lambda_{j}=1 \\
& \lambda_{j} \geq 0 \text { for all } j \in H .
\end{aligned}
$$

and basically proceed as in section 3 .

\section{Final Remarks}

Considering practical application of the cost allocation rules it may be convenient to eliminate dominated observations before formulating the parametric programming procedure in (3) to (6), simply in order to reduce the dimension of the problem. This may, for example, be done quite efficiently using DEA to partition the data set into efficient and inefficient observations.

The above approach has been outlined in a data envelopment framework due to the underlying natural assumptions about free disposability and convexity. Observe, however, that the programming problem stated in (3) to (6) with $t=1$ has the exact form of the master problem in the Dantzig-Wolfe decomposition procedure in linear programming. See for example Bazaraa et al. [4]. This procedure is valid for any linear programming problem which may naturally be split in two or more constraint sets. As discussed in the current DEA setting the allocation procedure technically transfers the dual variable corresponding to the convexity constraint onto the remaining constraints. Via the Dantzig-Wolfe decomposition (or directly) the current approach can be extended to transfer the marginal values of any selected constraints in a linear programming problem to the remaining constraints.

\section{References}

[1] Aumann, R and L. Shapley (1974), Values of Non-atomic Games, Princeton University Press.

[2] Banker R.D., A. Charnes and W.W. Cooper (1984), Some models for estimating technical and scale inefficiencies in Data Envelopment Analysis, Management Science, 30, 1078-1092.

[3] Banker R.D., Maindiratta, A (1988), Nonparametric analysis of technical and allocative efficiencies in production, Econometrica, 56, 13151332.

[4] Bazaraa, M.S., J.J. Jarvis and H.D. Sherali (1990), Linear Programming and Network Flows (2'ed.), Wiley. 
[5] Beasley, J.E. (2003), Allocating fixed costs and resources via data envelopment analysis, European Journal of Operational Research, 147, 198216.

[6] Billera, L.J. and C. Heath (1982), allocation of shared costs: a set of axioms yielding a unique procedure, Mathematics of Operations Research, 7, 32-39.

[7] Billera, L.J., C. Heath and J. Raanan (1978), Internal telephone billing rates - a novel application of non-atomic game theory, Operations Research, 26, 956-965.

[8] Bogetoft, P. (1996), DEA on relaxed convexity assumptions, Management Science, 42, 457-465.

[9] Bogetoft, P. J.M. Tama and J. Tind (2000), Convex input and output projections of nonconvex production possibility sets, Management Science, 46, 858-869.

[10] Charnes, A. W.W. Cooper and E. Rhodes (1978), Measuring the efficiency of decision making units, European Journal of Operational Research, 2, 429-444.

[11] Cooper, W.W., L.M. Seiford and J. Zhu (2004), Handbook on Data Envelopment Analysis, Kluwer Academic Publishers, Boston.

[12] Cook, W.D. and M. Kress (1999), Characterizing an equitable allocation of shared costs: A DEA approach, European Journal of Operational Research, 119, 652-661.

[13] Cook, W.D. and J. Zhu (2005), Allocation of shared costs among decision making units: a DEA approach, Computers and Operations Research, 32, 2171-2178.

[14] Friedman E. (2003), Paths and consistency in additive cost sharing, Economic Theory, 23, 643-658.

[15] Friedman, E. and H. Moulin (1999), Three methods to share joint costs or surplus, Journal of economic Theory, 87, 275-312.

[16] Mirman, L. and Y. Tauman (1982), Demand compatible equitable cost sharing prices, Mathematics of Operations Research, 7, 40-56. 
[17] Samet, D., Y. Tauman and I. Zang (1984), An application of the Aumann-Shapley prices for cost allocation in transportation problems, Mathematics of Operations Research, 9, 25-42. 